heights in the atmosphere. Typically $\Delta T$ was approximately 2-3 deg. C. a foot or so above the ground, 1 deg. at $10 \mathrm{ft}$., $0 \cdot 2 \mathrm{deg}$. at $55 \mathrm{ft}$. and, $0 \cdot 1 \mathrm{deg}$. at $180 \mathrm{ft}$., with a resolving time of about 1 sec., these results according with those of various micrometeorologists. A few preliminary observations made in Sydney of deviations produced in horizontal light beams at various heights were described by Dr. Giovanelli, the root mean square deviations falling off exponentially in the first $20 \mathrm{ft}$. with a scale-height of about $11 \mathrm{ft}$. These scanty results had been used to fix the height of the axis of the 5-in. equatorial photoheliograph at $9 \mathrm{ft}$. Mounted in the open, the use of air suction devices near all surfaces of this instrument exposed to direct sunlight, including the prime focus, seems to have eliminated completely any air inhomogeneities in or immediately outside the telescope.

Prof. Kiepenheuer showed a model of a new photoheliograph, to be made by Zeiss (Oberkochen), with the objective $10-14 \mathrm{~m}$. from the ground. This instrument is also to be mounted in the open, but a special feature is a completely separate wind shield surrounding the telescope tube. To reduce heating, the supporting tower is to be faced with white tile, the heat reflectance of which is reputedly extremely high. A rather different approach to telescope design is the new Pic-du-Midi instrument, where Dr. J. Rösch has the telescope tube projecting well through the slit of a dome, the slit being sealed to prevent the mixing of internal and external air. Whatever be the relative merits of these various schemes, it is clear that, by bold but careful planning, considerable progress has been made in reducing or eliminating internal seeing problems. Moreover, it was welcome to hear from Prof. Menzel that proper thermal insula. tion of the Iarge Sacramento Peak spectrograph had enabled it to yield definition as good as that of the vacuum spectrograph.

Whatever improvements are made, ground-based observations will almost certainly remain limited by atmospheric seeing conditions. Two devices to assist in the best use of moments of excellent seeing are the seeing monitor, which automatically selects these instants, and the electronic camera, which enables exposure times to be reduced so as to use them best. In Sydney, a seeing monitor operating on the steadiness of the solar limb has been used successfully by Dr. Bray and Mr. Loughhead to obtain, among other things, sequences of observations of facular granules; while a second device developed by Dr. Ramsay and $\mathrm{Mr}$. Kobler is ready for field trials on stellar images, and with slight modification can be used for deciding movements of greatest granule contrast. Dr. Rösch has been experimenting with a somewhat similar device, recording the intensity fluctuations as a pinhole is scanned rapidly across the solar image; even at the best of times, successive scans show significant differences due to seeing.

Photographs have been obtained by Prof. G. Wlerick (Observatoire de Meudon, Paris) and Dr. Rösch and Mlle. Dupré on nuclear emulsions with a Lallemand electronic camera. The great speed (approximately 100 times that of normal photography) has already been used to obtain improved photographs of double stars and planets, while high-resolution solar spectra have been photographed in less than $0 \cdot 1$ sec. There is little doubt that, combined with a seeing monitor, the electronic comera will yield spectra showing the full angular resolution of the telescope.

A group of papers on meteorological aspects of turbulence left one with the feeling that the association between solar seeing and turbulence has been given much too little attention; the field is wide open for exploration. This was the general feeling of participants also, who at the final session passed the following resolution: "The members of the symposium are convinced of the necessity for more extensive research on seeing problems, particularly with the view of application to the selection of observatory sites; and therefore recommend that basic studies be made of the optical properties of the atmosphere". R. G. GrovanELLI

\title{
TROPICAL ECOLOGY IN INDIA
}

A

SYMPOSIUM on "Ecological Problems in the Tropics" was held at Allahabad during February $3-5$, under the auspices of the National Academy of Sciences (India). The meetings were attended by more than one hundred scientists from all parts of the country. Dr. M. S. Randhawa, vice-president of the Indian Council of Agricultural Research, New Delhi, inaugurated the symposium and in his opening address specifically stressed the importance of ecological research in various development projects that the Government of India has taken in hand for the proper survey, development and exploitation of natural plant wealth of the country and for the increase in the production of food, fodder, fuel and fertilizers.

Dr. G. S. Pui, director of the Central Botanical Laboratory, Allahabad, who had organized the symposium, pointed out the progress so far made in Indian ecology and the need for a co-ordinated research programme in ecological studies in the tropics. He emphasized that the vegetation and animal life in the tropics raised problems entirely peculiar to these climetic conditions. He explained the concept of the forest ecosystem and recommended the use of The Forest Ecosystem Proforma published by the International Society for Tropical Ecology for use by the scientists in ecological studies and the application of the data to agriculture, forestry, soil and water conservation, desert immobilization, flood control, land use planning and wild life conservation, etc.

Forty-five papers were contributed to the symposium.

The paper by Sir Harry Champion on tropical ecological problems and by J. H. Davis were discussed with a number of those present. Prof. N. R. Dhar spoke on the value of nitrogen and phosphorus in Indian soils. Interesting papers were presented on the following subjects: tropical soils by Dr. G. S. Puri and K. Lakshminarayanan; fish ecology by Dr. S. M. Das and D. B. Saxena; arid zones by Sri S. K. Jain; relationships between habitat conditions and genome by Dr. R. P. Patil; distribution of perennial plants by Dr. J. K. Choudhury; soils of Madhya Pradesh by Dr. S. C. Pandeya; grasslands by Dr. S. S. Ramam; mangroves by Sri $\mathbf{S}$. S. Sidhu; ecological problems in 
the Western Himalayas by Sri M. K. Wali ; Aspergilli by Dr. B. S. Mehrotra; humid tropies by B. S. Ahuja and K. P. Singh; sal leaf litter by Sri N. K. Jain.

At the end of the symposium the following recommendations were made:

"Ecologists working on the tropical region, particularly Indian ecologists, should undertake co-operative studies to fill up the lacunæ in our knowledge as soon as possible. For achieving the purpose it is desirable to launch a vigorous and intensive programme of research with a view to obtaining data of practical and immediate applicability to agriculture, forestry and soil, water and wild life conservation. It is felt that The Forest Ecosystem Proforma prepared and pub- lished by the International Society for Tropical Ecology will be found of extensive utility. The same with a suitable amendment can be conveniently used for studies of animal and human ecology."

A committee to implement and expand the results of the symposium and to raise necessary funds needed for supporting the project in co-operation with the International Society for Tropical Ecology was formed. Further information concerning this project can be obtained from Dr. G. S. Puri, general secretary, International Society for Tropical Ecology, 10 Chatham Lines, Allahabad. The Forest Ecosystem Proforma can be obtained from the Society, price Rs. 5.

\section{THE WEST AFRICAN RICE RESEARCH STATION}

$\mathrm{T}$ HE West African Rice Research Station, which is situated at Rokupr in Sierra Leone, is supported by the four West African territories of Nigeria, Ghana, Sierra Leone and Gambia. It is now ten years since a free grant from the United Kingdom under the Colonial Development and Welfare Act enabled the existing experimental station of the Sierra Leone Department of Agriculture to be transformed into a major inter-territorial research station, and five years since completion of buildings and establishment enabled a full-scale research programme to be started. It is hoped that this year will mark a further stage in development and that the Station will attain the status of an independent institute.

Rice production and consumption is expanding rapidly in West Africa, not only in Sierra Leone and adjacent parts where it has long been the staple food of the population but also in all other territories where it is of more recent cultivation. Present production in the whole of West Africa is estimated to be about one and a half million tons of paddy per annum, of which just more than a quarter of a million tons is produced in Sierra Leone. In view of the important part that rice seems destined to play in the economy of West Africa as a major food crop and in efficient utilization of the land, all Governments gave support to the establishment of the Station, the basic policy of which is to carry out fundamental research on rice as a crop under West African conditions, and to advise territorial Ministries, who remain responsible for local application and extension work, on their programmes of rice development and production.

The main station at Rokupr, in addition to an area of upland for administrative, laboratory and farm buildings and staff quarters and for experimental work on upland rices, consists of a 66-acre swamp rice-farm contiguous with the estuary of the Great Scarcies River, from which it is irrigated by tidal flooding with fresh water throughout the growing season. In addition, the Station maintains small areas on other types of soil in different parts of Sierra Leone. The laboratories, which were built in 1955 , are well equipped and have recently been supplemented by a series of cabinets with controlled illumination and temperature for work on growth, an opensided greenhouse for pot-work with soils and culture media, and a deep-water tank for experimental work with floating and flood-resistant rices. An airconditioned building is provided for storage of the seed of the West African varietal collection, which is maintained by the Station, the responsibilities of which also include the maintenance of nucleus pure seed stocks of established and recommended varieties for seed multiplication schemes of the contributing territories.

At present the cultivation of rice in West Africa is carried out under a wide range of notural water conditions, from those of coastal mangrove swamps to the seasonal flooding of riverain plains in the lower-rainfall areas. There are extensive and widespread areas, potentially useful for rice growing, at present undeveloped. The attention of the Station has thus been concentrated on the investigation of the rice plant in relation to its habitat and on the production of rice varieties to suit natural conditions, together with work on soils and the changes that occur in them when modification of conditions may take place during development.

Selection from local and introduced varieties of rice has given excellent results in the improvement of yields in the past, but the stage has been reached when further advances can only result from a breeding programme which includes hybridization. The primary object of the present programme is the provision of high-yielding acceptable rice varieties for all the major West African habitats, including deep-flooding areas and rain-fed uplands. While consideration is given to factors such as diseaseresistance, straw-strength and milling quality, none of these at present is of such importance as to warrant specific breeding programmes, and they are considered merely as they reflect on yield. Recent series of trials in various parts of West Africa indicate, however, that in some areas effects of light and, possibly, temperature are limiting in preventing existing established varieties from attaining their full potential, and a specific breeding programme for early maturing varieties insensitive to photoperiod is being undertaken. In conjunction, physiological investigations have been started on the effects of light and temperature on duration and yield.

Other physiological work to date has been largely designed to produce useful techniques for the plant breeders. Work on viability and dormancy has resulted in improved storage of seed and speeding up the breeding programme, while investigations into hydroponics and culture media have led to a method of clonal multiplication of considerable value in experimental use. Work on milling quality-in particular with the use of parboiling, the recommended and accepted method in West Africa-has indicated 